\title{
Symptomatic Cytomegalovirus Enteritis in an Immunocompetent Individual
}

\author{
Elizabeth D. Krebs, ${ }^{1}$ Robin T. Petroze, ${ }^{2}$ Sarah M. Kelting, and Zequan Yang ${ }^{1}$
}

\begin{abstract}
Background: Cytomegalovirus most commonly affects immunocompromised individuals. However, it is also a rarely reported cause of enteritis and colitis in immunocompetent patients. Occasional case reports associate the infection with bowel ischemia in this population.

Case Presentation: A 75-year-old African American male with no history of immunosuppression presented with three weeks of abdominal pain, nausea, and vomiting. Computed tomography and physical examination were concerning for mesenteric ischemia from superior mesenteric artery and superior mesenteric vein thrombosis, and the patient underwent operative small bowel resection. Pathologic evaluation revealed cytomegalovirus infection rather than ischemic changes. The patient was treated subsequently with ganciclovir and recovered well.

Conclusion: Cytomegalovirus enterocolitis should be considered even in immunocompetent individuals. Its association with mesenteric and colonic ischemia is unclear. When identified, cytomegalovirus infection can be treated with appropriate antiviral medication with good results.
\end{abstract}

Keywords: cytomegalovirus; ischemia; enteritis; immunocompetent

C Ytomegalovirus (CMV) is a herpes virus known to cause symptomatic colitis and enteritis in immunocompromised individuals. In immunocompetent individuals, however, CMV infection is usually asymptomatic or presents as a mononucleosis-type syndrome [1]. Few case reports describe CMV infection in immunocompetent individuals, with even fewer reporting enteritis from CMV infection. Recognition of these cases is important, as CMV represents a rarely tested yet well treated cause of enteritis.

We present the case of a 75-year-old immunocompetent individual who underwent exploratory laparotomy and small bowel resection for presumed mesenteric ischemia. However, pathologic evaluation revealed CMV enteritis. He was subsequently treated with antiviral medication and recovered appropriately.

\section{Case Presentation}

A 75-year-old African American male with a history of deep vein thrombosis, non-ischemic cardiomyopathy, gout, and hypertension presented to our institution from an outside hospital with a three-week history of abdominal pain, nausea, and vomiting. Original computed tomography (CT) scans were significant only for circumferential thickening of the distal small bowel. The patient had completed a course of ciprofloxacin and metronidazole with no change in condition, with stool studies negative for community pathogens and Clostridium difficile. He had no notable travel history, sick contacts, hypotensive episodes, or additional symptoms. Colonoscopy prior to transfer revealed focal active colitis. Because of the patient's continued pain, he underwent computed tomography angiography (CTA) that revealed thrombosis of the superior mesenteric artery (SMA) and superior mesenteric vein (SMV), with thickening of the terminal ileum and right colon as well as dilated small bowel. At this time, he was transferred to our institution.

At our hospital, the patient was afebrile with no leukocytosis or lactic acidosis. His abdominal examination was benign with no tenderness or distension. He was started on therapeutic anticoagulation and monitored with serial abdominal

\footnotetext{
${ }^{1}$ Department of Surgery, ${ }^{3}$ Department of Pathology, University of Virginia, Charlottesville, Virginia.

${ }^{2}$ Division of Pediatric General and Thoracic Surgery, Montreal Children's Hospital, 1001 Boulevard Décarie, Montréal, Quebec, Canada.

(C) Elizabeth D. Krebs et al. 2017; Published by Mary Ann Liebert, Inc. This Open Access article is distributed under the terms of the Creative Commons License (http://creativecommons.org/licenses/by/4.0), which permits unrestricted use, distribution, and reproduction in any medium, provided the original work is properly credited.
} 


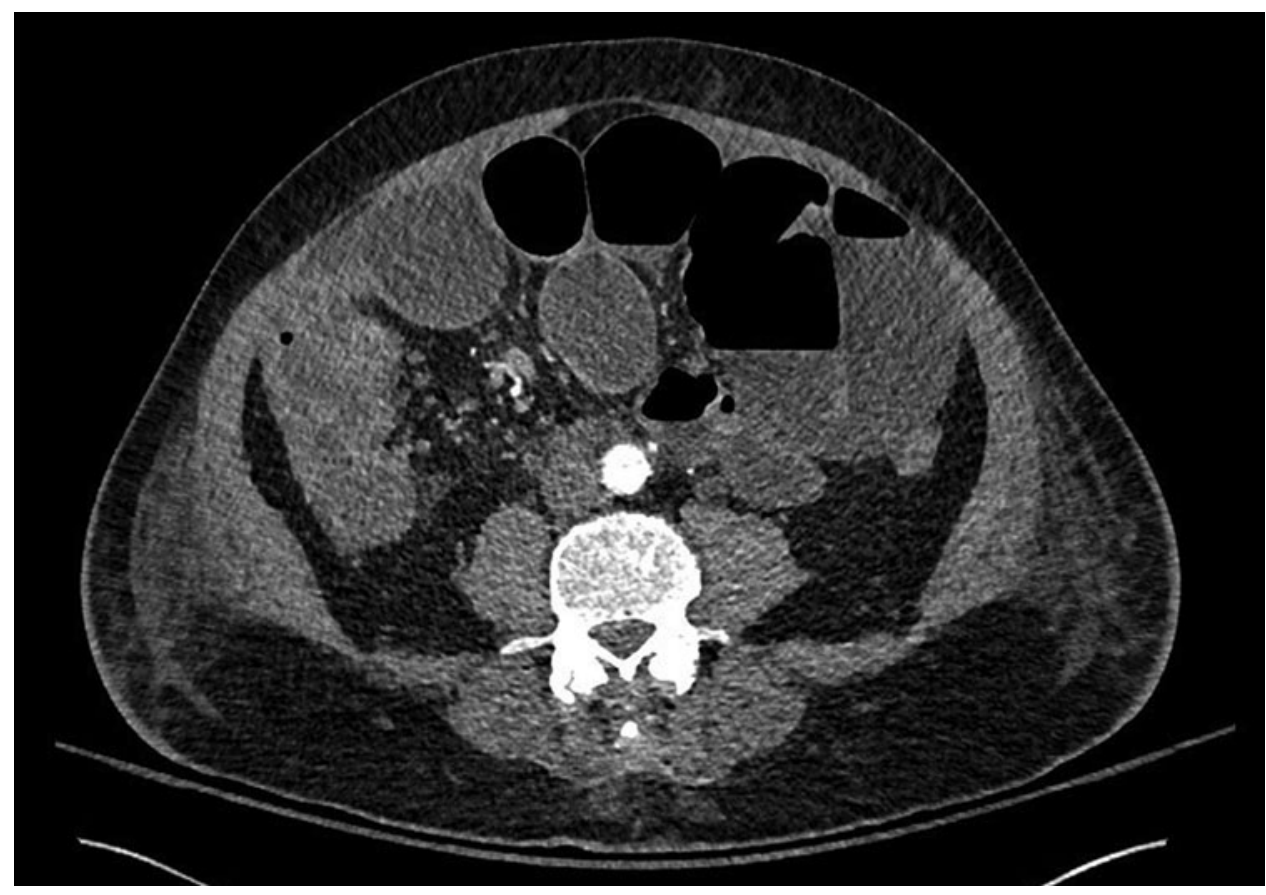

FIG. 1. Computerized tomography scan from admission revealing decompressed terminal ileum and small bowel inflammation.

examinations and lactic acids. On hospital day two he had increased abdominal pain as well as distension, tympany, and tenderness to palpation on examination. Repeat CTA at this time revealed worsening inflammation of the distal ileum, cecum, and proximal ascending colon, as well as evolving SMV thrombosis (Fig. 1). He was taken to the operating room for exploratory laparotomy.

During laparotomy, the small bowel was found to be diffusely edematous with extensive dilation. The ileum was particularly distended and congested, with the terminal $100 \mathrm{~cm}$ appearing non-viable without reliable blood flow on Doppler examination. However, the cecum and entire colon were grossly normal. The patient had a palpable SMA pulse and strong Doppler signals along secondary mesenteric ves- sels. A total of $105 \mathrm{~cm}$ of distal ileum were resected, and the patient was left with an open abdomen in discontinuity. He was taken back to the operating room for a second look on post-operative day one, where remaining bowel was viable. His bowel was returned to continuity and his abdomen was closed. His subsequent recovery was remarkable only for profuse watery diarrhea.

On gross and microscopic pathology, the ileal specimen had no definitive features of ischemic injury. Surprisingly, intra-nuclear and intra-cytoplasmic inclusions were seen, suggestive of CMV infection (Fig. 2, left) [2]. The specimen was then investigated with immunohistochemical stain for CMV (Fig. 2, right) and was found to be positive. The patient was tested subsequently and found to have an elevated CMV

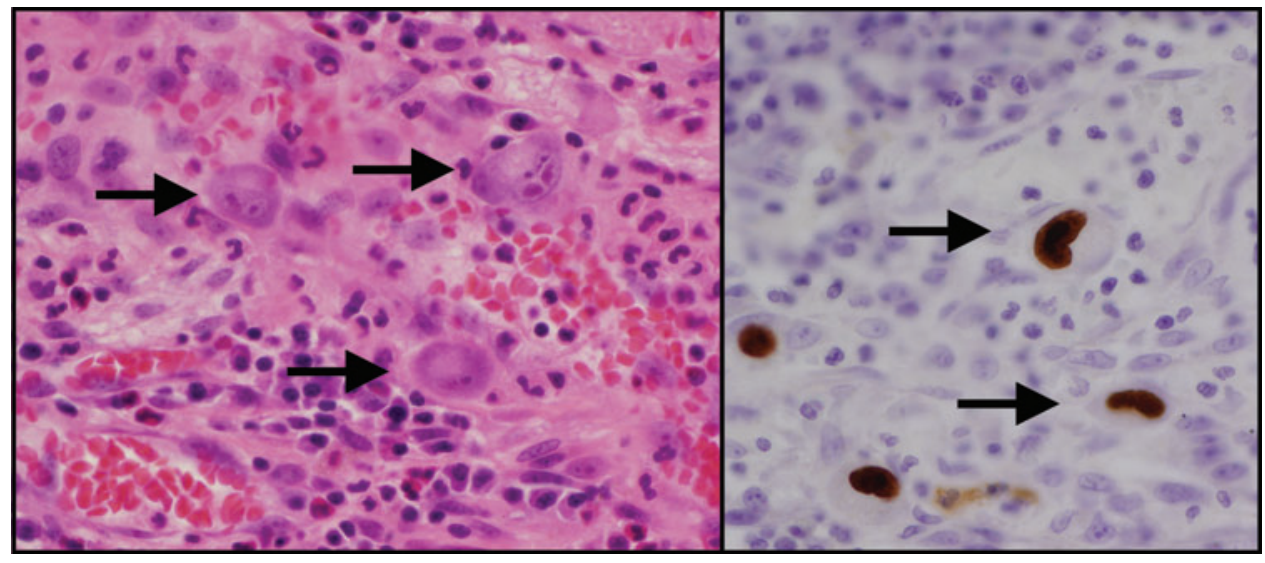

FIG. 2. Left: High-power field of a hematoxylin and eosin stained section of the small bowel resection specimen. Infected cells demonstrate classic cytomegalovirus (CMV)-associated viral cytopathic effect, including enlarged cells with smudgy intra-nuclear inclusions surrounded by a clear halo and granular intra-cytoplasmic inclusions (arrows). Right: An immunohistochemical stain for CMV is positive in the intra-nuclear inclusions, as highlighted by the brown chromogen (arrows). 
viral load, with positive immunoglobulin $\mathrm{M}$ and $\mathrm{G}$ antibodies, indicating a primary CMV infection.

The patient was treated with a six-week course of valgancyclovir as well as therapeutic anticoagulation. He has continued to recover well. Given the concern for immunosuppressed state, he was tested for human immunodeficiency virus (HIV) and CD4 count, which were negative and within normal limits. He also underwent a malignant workup, including full-body CT scan, which was negative. His abdominal pain, distension, and diarrhea have resolved after therapy for his CMV enteritis.

\section{Discussion}

Cytomegalovirus is a double-stranded DNA virus in the herpes virus family. Infections occur as primary infection or re-activation of latent virus and can involve the gastrointestinal tract, respiratory tract, central nervous system, and retina [3]. Although worldwide seropositivity is 40\%-100\% [1], symptomatic infections are most common in patients immunocompromised from HIV, chemotherapy, or after transplant.

Cytomegalovirus enteritis and CMV colitis are reported rarely in immunocompetent individuals. A systematic literature review performed by Karigane et al. [4] revealed only 32 reported cases of CMV enteritis or colitis in immunocompetent individuals from 1990-2012. The most common symptom was diarrhea $(76 \%)$, followed by abdominal pain (52\%) The median age was 70 , with $75 \%$ of patients older than 60, indicating that CMV enteritis may be more common in elderly patients.

Our literature search revealed only two case reports of CMV colitis or enteritis associated with ischemia in an immunocompetent individual. Naseem et al. [3] reported a case of ileal ischemia and CMV, in which a patient presented similarly to ours, with abdominal distension and thickened distal ileum on CT scan, and underwent open hemicolectomy. Unlike our case, their patient's pathology revealed both cytoplasmic inclusions and segmental ischemic changes, indicative of both ischemia and CMV enteritis. Hasegawa et al. [5] reported a case of CMV colitis associated with ischemic colitis. In their report, an 85-year-old patient presented with diarrhea, with ischemic colitis diagnosed by colonoscopy. Twenty days later, after symptoms continued, repeat colonoscopy revealed CMV viral inclusions, which was treated with valgancyclovir.

In these cases and our own, the relation between CMV and ischemia is not clear. It is possible the thrombosed vessels predated the CMV, creating a chronic ischemic state that predisposed to infection. It is also possible that CMV leads to a prothrombotic state, leading to the thromboses. Cytomegalovirus has been associated in case reports with thrombosis at various sites, including splanchnic vein thrombosis and deep vein thrombosis (DVT)/pulmonary embolism (PE) [6]. This association has been studied in one case control study of 140 patients diagnosed with CMV, which found thrombosis in $6 \%$ of patients, and found CMV infection to be independently associated with thrombosis [7]. However, this is the only published investigational study on the topic. In our case, as there were no ischemic changes found in the diseased small bowel, the SMA/SMV thrombosis and distal ileal enteritis likely resulted from systemic CMV infection but have no cause-effect relation.

\section{Conclusion}

Cytomegalovirus should be recognized as a cause of enteritis even in immunocompetent patients. It should be considered if the common etiologies are ruled out. When it is identified and treated appropriately with antiviral medication, patients can recover well from CMV enteritis and avoid surgical intervention.

\section{Author Disclosure Statement}

No competing financial interests exist.

\section{References}

1. de la Hoz RE, Stephens G, Sherlock C. Diagnosis and treatment approaches of CMV infections in adult patients. J Clin Virol 2002;25(Suppl 2):S1-12.

2. Goodman AL, Murray CD, Watkins J, et al. CMV in the gut: A critical review of CMV detection in the immunocompetent host with colitis. Eur J Clin Microbiol Infect Dis 2015;34:13-18.

3. Naseem Z, Hendahewa R, Mustaev M, et al. Cytomegalovirus enteritis with ischemia in an immunocompetent patient: A rare case report. Int J Surg Case Rep 2015;15:146-148.

4. Karigane D, Takaya S, Seki Y, et al. Cytomegalovirus enteritis in immunocompetent subjects: A case report and review of the literature. J Infect Chemother 2014;20:325-329.

5. Hasegawa T, Aomatsu K, Nakamura M, et al. Cytomegalovirus colitis followed by ischemic colitis in a non-immunocompromised adult: A case report. World J Gastroenterol 2015;21:3750-3754.

6. Justo D, Finn T, Atzmony L, et al. Thrombosis associated with acute cytomegalovirus infection: A meta-analysis. Eur J Intern Med 2011;22:195-199.

7. Atzmony L, Halutz O, Avidor B, et al. Incidence of cytomegalovirus-associated thrombosis and its risk factors: A case-control study. Thromb Res 2010;126:e439-443.

Address correspondence to: Dr. Elizabeth D. Krebs Department of Surgery University of Virginia PO Box 800136

Charlottesville, VA 22908

E-mail: edk9f@virginia.edu

\begin{aligned} & \multicolumn{1}{c|}{ Abbreviations Used } \\ $\mathrm{CMV} & =$ cytomegalovirus \\ $\mathrm{CT} & =$ computed tomography \\ $\mathrm{CTA} & =$ computed tomography angiography \\ $\mathrm{DVT} & =$ deep vein thrombosis \\ $\mathrm{HIV} & =$ human immunodeficiency virus \\ $\mathrm{PE} & =$ pulmonary embolism \\ $\mathrm{SMA} & =$ superior mesenteric artery \\ $\mathrm{SMV} & =$ superior mesenteric vein \end{aligned}

Cite this article as: Krebs ED, Petroze RT, Kelting SM, Yang Z (2017) Symptomatic cytomegalovirus enteritis in an immunocompetent individual. Surgical Infections Case Reports 2:1, 23-25, DOI: 10.1089/crsi.2017.0004 